\title{
Dual Variable Domain Immunoglobulin ABT-165
}

National Cancer Institute

\section{Source}

National Cancer Institute. Dual Variable Domain Immunoglobulin ABT-165. NCI

Thesaurus. Code C116750.

A dual-specific, tetravalent immunoglobulin (Ig) G-like molecule targeting two as of yet not publicly known targets, with potential antineoplastic activity. The target-binding variable domains of two monoclonal antibodies, which are not publicly known, are combined, via linkers, to create the tetravalent, dual-targeting single agent ABT-165. Upon administration of dual variable domain immunog lobulin (DVD-Ig) ABT-165, the target-binding variable domains specifically recognize and simultaneously bind to their two antigens expressed on tumor cells. This may both prevent antigen-mediated signaling and lead to an inhibition of cellular proliferation in susceptible tumor cells. The antigen targets are overexpressed on certain tumor cell types. The DVD-Ig may have enhanced physicochemical and pharmacokinetic properties as compared to their antibody counterparts. 